\title{
Lenalidomide-Induced Hypersensitivity Pneumonitis
}

\author{
Martin Kortuem Hermann Einsele \\ Department of Internal Medicine II, Division of Hematology and Medical Oncology, University Hospital, Würzburg, Germany
}

The introduction of the so-called novel agents - bortezomib, thalidomide and lenalidomide - has significantly improved the outcome of multiple myeloma treatment. Pneumonitis-like side effects occurring during treatment with novel agents are rare but have been described following application of the first-in-class proteasome inhibitor bortezomib as well as for the immunomodulator thalidomide, but had not yet been described for its successor lenalidomide.

The myeloma patient described by Lerch et al. [1] developing pneumonitis-like symptoms after 2 weeks of treatment with lenalidomide monotherapy is the second case report of lenalidomide induced pulmonary hypersensitivity reaction in literature - assuming lenalidomide as the causative agent. Interestingly, the described patient had previously received the lenalidomide predecessor thalidomide with dexamethasone without showing any pulmonary side effects. The first case report was described in 2007 by Thornburg et al. [2] where a 60 -year-old female myeloma patient developed signs of a hypersensitivity pneumonitis-like reaction after receiving lenalidomide and dexamethasone therapy. The symptoms occurred after she had already received 2 cycles of therapy; symptoms were potentially delayed by the intense, concomitant high-dose steroid treatment. In contrast a 58-year-old, male myeloma patient in our center, receiving just a short course of steroid treatment in a lenalidomide, adriamycin, and dexamethasone therapy in 2008, showed a sudden onset, developing pulmonary infiltrates and requiring hospitalization. This happened shortly after he had finished dexamethasone therapy and after had been on lenalidomide therapy for only a few days. Lenalidomide therapy was stopped and antibiotic treatment was started which led to a slow recovery. After he had fully recovered from the pulmonary symptoms the second course of lenalidomide therapy was started, leading again to sudden pneumonitis-like symptoms. This time broncho-alveolar lavage was performed without any documentation of bacterial, viral or fungal infection, a lenalidomide induced pulmonary drug reaction was assumed. After treatment with steroids and intravenous antibiotics, pulmonary symptoms recovered within few days. The transbronchial biopsies in the patients described by Lerch et al. [1] and Thornburg et al. [2] revealed a nonspecific, hypersensitivity pneumonitis (compatible with toxic drug-induced damage). Immunosuppressive therapy with steroids induced a rapid relief of all symptoms in all 3 cases. Infectious cause of the pulmonary infiltrates could be excluded by broncho-alveolar lavage. No eosinophilia was found, as reported in literature in several cases of thalidomide induced pulmonary reactions. Taking the well-tolerated thalidomide pretreatment of the here described patient in consideration, rather a drug-, than a class-specific adverse effect may be suggested. Accordingly, Pretz et al. in 2009 [3] described a 76-year-old female myeloma patient who had developed a thalidomide induced pneumonitis and showed a good tolerance to a subsequent lenalidomide therapy.

As described for bortezomib and thalidomide, lenalidomide therapy may cause severe pulmonary side effects in rare cases. The pathomechanism remains unclear, a toxic or immunologically mediated mechanism can be envisaged. Physicians prescribing lenalidomide should be aware of this rare but severe pulmonary complication, and should consider a lenalidomide induced hypersensitivity pneumonitis if patients develop a pulmonary affection during lenalidomide therapy. Especially in patients not responding to antibiotic therapy a broncho-alveolar lavage and if possible a transbronchial biopsy are recommended to document a hypersensitivity reaction which should be treated by steroids and to exclude an infectious disease. In these patients further exposition to lenalidomide may be harmful, potential alternatives should be considered.

\section{Conflict of Interest}

M.K.: No competing interests to declare.

H.E.: Honoraria: Celgene Germany; Ortho Biotech, Germany. Consultancy: Celgene; Ortho Biotech. Research funding: Ortho Biotech.

\section{References}

1 Lerch E, Györik S, Feilchenfeldt J, Mazzucchelli L, Quadri F: A Case of Lenalidomide-Induced Hypersensitivity Pneumonitis. Onkologe 2010;33: 249-252.
Thornburg A, Abonour R, Smith P, Knox K, Twigg HL III: Hypersensitivity pneumonitis-like syndrome associated with the use of lenalidomide. Chest 2007;131:1572-1574.
3 Pretz J, Medeiros BC: Thalidomide-induced pneumonitis in a patient with plasma cell leukemia: no recurrence with subsequent lenalidomide therapy. Am J Hematol 2009;84:698-699.

\section{KARGER}

Fax +497614520714

Information@Karger.de

www.karger.com (c) 2010 S. Karger GmbH, Freiburg

Accessible online at:

www.karger.com/onk
Dr. med. Martin Kortüm

Universitätsklinikum Würzburg

Medizinische Klinik und Poliklinik II

Oberdürrbacher Str. 6, 97080 Würzburg, Germany

Tel. +49 931 201-40001, Fax -640001

Kortuem_m@klinik.uni-wuerzburg.de 